\title{
A Preliminary Numerical Investigation of Airborne Droplet Dispersion in Aircraft Cabins
}

\author{
Dinesh Bhatia ${ }^{1}$, Antonio De Santis ${ }^{2}$ \\ ${ }^{1}$ School of Aerospace, University of Nottingham Ningbo China, Ningbo, China \\ ${ }^{2}$ Department of Aeronautical Engineering, Military Technological College, Muscat, Oman \\ Email: Dinesh.Bhatia@nottingham.edu.cn
}

How to cite this paper: Bhatia, D. and De Santis, A. (2020) A Preliminary Numerical Investigation of Airborne Droplet Dispersion in Aircraft Cabins. Open Journal of Fluid Dynamics, 10, 198-207. https://doi.org/10.4236/ojfd.2020.103013

Received: June 30, 2020

Accepted: July 31, 2020

Published: August 3, 2020

Copyright (c) 2020 by author(s) and Scientific Research Publishing Inc. This work is licensed under the Creative Commons Attribution International License (CC BY 4.0).

http://creativecommons.org/licenses/by/4.0/

\section{(c) (i) Open Access}

\begin{abstract}
The emergence of the novel coronavirus has led to a global pandemic which has led to the airline industry facing severe losses. For air travel to recover, airlines need to ensure safe air travel. In this paper, the authors have modeled droplet dispersion after a single breath from an index patient. Computational Fluid Dynamics (CFD) simulations are conducted using the k- $\omega S S T$ turbulence model in ANSYS Fluent. The authors have taken into consideration several parameters such as the size of the mouth opening, the velocity of the cabin air as well as the number of droplets being exhaled by the index patient to ensure a realistic simulation. Preliminary results indicate that after a duration of $20 \mathrm{~s}$, droplets from the index patient disperse within a $10 \mathrm{~m}^{2}$ cabin area. About $75 \%$ of the droplets are found disperse for up to $2 \mathrm{~m}$ axially behind the index patient. This could possess an enhanced risk to passengers sitting behind the index patient. Ultimately, this paper provides an insight into the potential of CFD to visualise droplet dispersal and give impetus to ensure that necessary mitigating measures can be taken to reduce the risk of infection through droplet dispersal.
\end{abstract}

\section{Keywords}

Droplet Dispersal, Aircraft Cabin, CFD, Airborne Transmission

\section{Introduction}

The global pandemic due to COVID-19 has affected the day to day life of billions of people and has severely affected the world economy. COVID-19 is one of the many airborne infectious diseases such as influenzas, TB, and the flu that are affecting the global populace. Until the advent of the COVID-19 pandemic, air traffic was increasing at the rate of $4.4 \%$ year on year [1]. Global air traffic has since plunged $94.3 \%$ in April 2020 as compared to April 2019 and airlines are 
facing a potential loss of $\$ 252$ billion in 2020 [2] [3]. For the airline industry to recover, growth in air traffic is vital. However, it is necessary to forecast and moderate the risk of infection from airborne infectious diseases in aircraft cabins to enable air travel to recover to pre pandemic levels. A study of the droplet dispersion from the breath of a passenger in an aircraft cabin can provide a unique insight into mitigating measures that can be undertaken to ensure safe air travel. Modeling of this dispersion can enable the airline industry to take the necessary measures to ensure safe travel which will instil confidence amongst passengers and lead to the growth of air travel in the near future.

The dispersion of droplets (in the case of airborne infectious diseases) greatly depends on the airflow within the aircraft cabin. The humidity of the cabin air is the prevalent factor in the aircraft cabin that can impact the transmission of airborne diseases. The distance between people seated next to each other in an airplane with full passenger loads is approximately $0.5 \mathrm{~m}$ or less. In the confined space of an airplane cabin, and during long flights, direct dispersion is a potential pathway for airborne transmission of infectious diseases [4]. During air travel, some of the outbreaks of tuberculosis, SARS, influenza, are alleged to have happened [5]. The direct contact, indirect contact, airborne route, or droplets could carry on the transmission of the diseases. Therefore, it is essential to quantify the potential impact of droplets exhaled from passengers within the aircraft cabin to ensure that precautions can be taken for safe air travel.

There are a limited number of studies which have investigated the generation and dispersion of particles originated from human expiratory activities in aircraft environments as compared to the built environment. Yang et al. [6] conducted an experimental investigation using a Boeing 767 aircraft cabin to study the dispersion behaviour of expiratory droplets released from a coughing person. The investigation result shows that when the injection point was closer to the cabin wall i.e. the window section, the droplets dispersion was greatly suppressed. Similar results were obtained by Gupta et al. [7] through their numerical modeling of droplet dispersal when talking, coughing and breathing. Figure 1 shows the dispersal of droplets during a single breath by a person over a duration of $240 \mathrm{~s}$. Results presented by Gupta et al. indicate that the droplet dispersal due to a single breath reduces by $52 \%$ after 1 min (shown in Figure 1). Similar results were obtained during coughing by a person and resulted in an $88 \%$ reduction in droplets after 4 minutes although the volume of droplets in coughing is much higher. While the above studies focus on the rate of droplet dispersal, the proportion of droplets dispersed through a single breath reaching other passengers is not touched upon.

In this paper, the authors will build upon existing data from literature presented herein and conduct a Reynolds Averaged Navier Stokes (RANS) simulation of the droplet dispersal in an aircraft cabin using a commercial CFD package such as ANSYS Fluent. The data presented in this paper is based on realistic and accurate modelling of conditions within an existing cabin. The authors have 


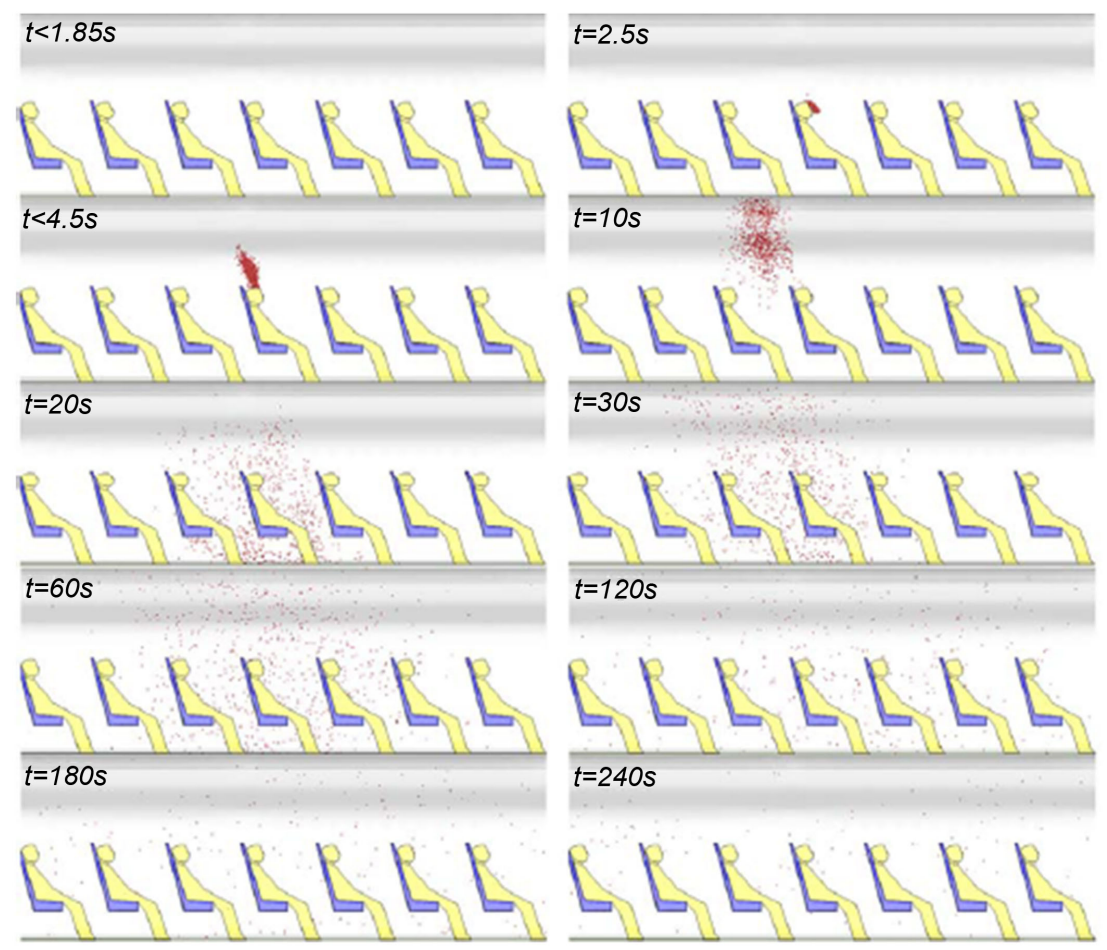

Figure 1. Droplet dispersal from a single breath for a total duration of $240 \mathrm{~s}$ as modeled by Gupta et al. [7].

simulated an actual average adult (the index patient) and have taken into consideration several factors including accurate mouth opening size during a single breath as well as the volume of air exhaled by an average adult in a minute. Apart from this, the authors have considered the actual air flow velocity from the aircraft ventilation system to present a realistic and accurate picture of droplet dispersal within the cabin. This paper aims to identify the critical area of influence of droplet dispersal after a single breath from the index patient and its impact on passengers within the cabin. The quantification and visualisation of this droplet dispersal can provide an insight to airline companies to identify potential precautions to ensure safe travel in the future.

\section{Methodology}

In Section 1 it was noted that the carrier of contagious agents may be the droplets exhaled by a passenger who might be the carrier of an infectious disease. Aircraft cabins being an indoor environment, may be highly susceptible to airborne contagious agents. The transmission of droplets exhaled by the index patient has been computed in the present investigation for a passenger who is seated in the middle of a seven-row, twin-aisle, fully occupied cabin using the FLUENT commercial CFD package. However, as existing studies show the range of droplet transmission to be $2 \mathrm{~m} \mathrm{[8]}$ and for quick and efficient results, the size of the cabin in ANSYS has been modeled to be of approximately $10 \mathrm{~m}^{2}$ (incorporating a $2 \mathrm{~m}$ distance in the front as well as the back of the index patient re- 
spectively). Apart from this, simulations were conducted for droplets exhaled from a single breath from the index patient.

This section is divided into two main subsections. Subsection 2.1 deals with the geometry for the model whereas Subsection 2.2 deals with the simulation setup as well as the details of the mesh.

\subsection{Geometry of the Model}

The geometry for the model involves the use of life-size human model with accurate geometrical dimensions of a real person. The mannequin/index patient was designed to have a height of $1.75 \mathrm{~m}$ and was placed in a seated position as shown in Figure 2. The mannequin was designed using the blender software and imported into ANSYS for mesh generation.

The mannequin was designed with two circular nostrils with a diameter of 11 $\mathrm{mm}$. The mouth opening was modeled to have an area of $120 \mathrm{~mm}^{2}$. To make the design as realistic as possible, the respiratory frequency of breathing was assumed to be $15.5 \mathrm{~min}^{-1}$. To ensure rapid and accurate results, the geometry was simplified once it was imported into ANSYS and several surfaces were combined for ease of mesh generation. The seat was also deleted from the simulation, mainly because the simulation focused on tracking the droplet dispersal and the seat would result in use of additional computer resources without influencing the results. Figure 3 shows the insertion of the mannequin into the actual fluid domain.

\subsection{Simulation Setup}

To ensure rapid and accurate results and to simplify the preliminary study, the authors considered a portion of the cabin that covers an area of $10 \mathrm{~m}^{2}$ around the passenger. An assumption was made on the size of the cabin taking into consideration the distance travelled by the droplets during a normal exhalation [9]. Due to the complex geometry of the mannequin, the authors decided to use an

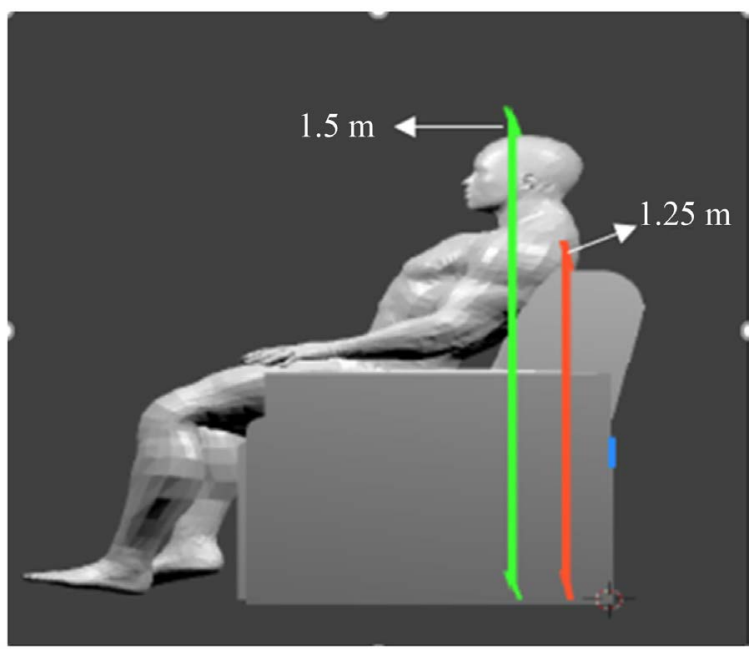

Figure 2. Mannequin design in a seated position. 


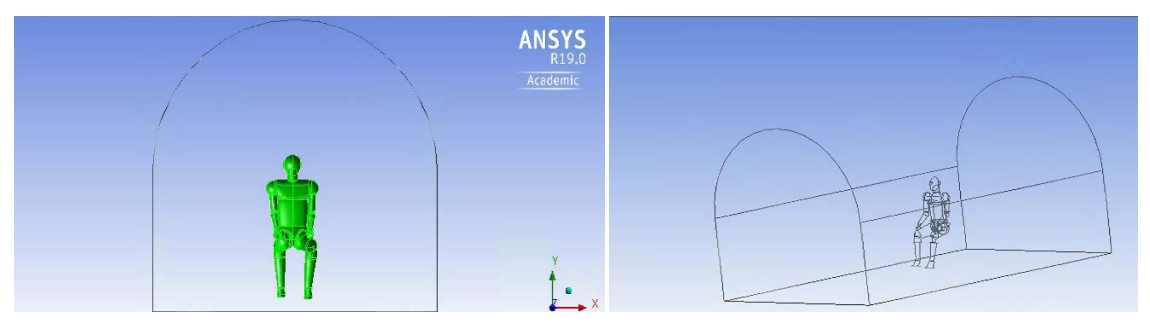

Figure 3. Insertion of the mannequin into ANSYS. The mannequin is assumed to be in the centre of the domain. The domain is assumed to have the following dimensions: $4 \mathrm{~m}$ (l), $2.5 \mathrm{~m} \mathrm{(b)} \mathrm{and} 2.5 \mathrm{~m} \mathrm{(h).}$

unstructured mesh with a total mesh size of $1.9 \times 10^{5}$ elements with a minimum element size of $0.3 \mathrm{~mm}$ (shown in Figure 4). The mesh size was determined by following the standard procedure for performing a mesh independence study in order to ensure a good balance between accuracy and computational processing capabilities [10]. The main purpose of this research is to track the path of the droplets in a large domain, with the index patient sitting in the middle of the domain. Therefore, the choice of the mesh size was dictated by the nature of the study which, focused on studying the central regions of the domain and allowed the authors to neglect the effect of the boundary layer further away from the index patient. However, the minimum element size in the present study $(0.3 \mathrm{~mm})$ was chosen to ensure that the finer details of the flow phenomenon were also captured.

To verify the interaction between a droplet coming out during a normal exhalation process and the aircraft ventilation system, a velocity inlet boundary condition was chosen at the top of location vertically above the seat. This closely represents the usual set up of an aircraft cabin. The inlet velocity was chosen from standard values within an aircraft cabin (shown in Table 1) and set at 0.7 $\mathrm{ms}^{-1}$ blowing downwards along the negative vertical axis at a temperature of $20^{\circ} \mathrm{C}$. The flow regime inside the cabin is a regime of forced convention. Considering a jet stream of air at $20^{\circ} \mathrm{C}$ with a velocity of $0.7 \mathrm{~ms}^{-1}$, the flow is found to be turbulent. The turbulence model chosen for the simulation is the k- $\omega S \mathrm{ST}$ model owing to its accuracy in predicting flow patterns and temperature stratification in an indoor environment [11]. To consider the effect of the temperature in the transport phenomenon, the energy component of the Navier Stokes and the gravity were taken into account for the calculation of the solution.

To ensure that the simulation was realistic, modeling of the exhalation was a very critical part of the simulation setup. An average human being exhales 525 droplets of water vapour in a single breath with each droplet being $0.4 \mu \mathrm{m}$ in diameter [12]. The duration of a single breath is approximately $1.5 \mathrm{~s}$. The act of exhaling in a normal condition for an average human being, in a relaxed environment was modeled as discrete phase in a multiphase flow (air and water vapour) with the injection of stochastic number of inert particles with an average diameter of $100 \mu \mathrm{m}$ so as to enable the droplets to interact with the surrounding fluid. The velocity of the droplets depends on the diameter but for the purpose 
Table 1. Average cabin air circulation velocities at different locations within the cabin [7].

\begin{tabular}{cc}
\hline Location & Air Velocity $\left(\mathrm{ms}^{-1}\right)$ \\
\hline Window zone & 0.35 \\
Top of the cabin & 0.7 \\
Centre of the cabin & 0.25 \\
\hline
\end{tabular}
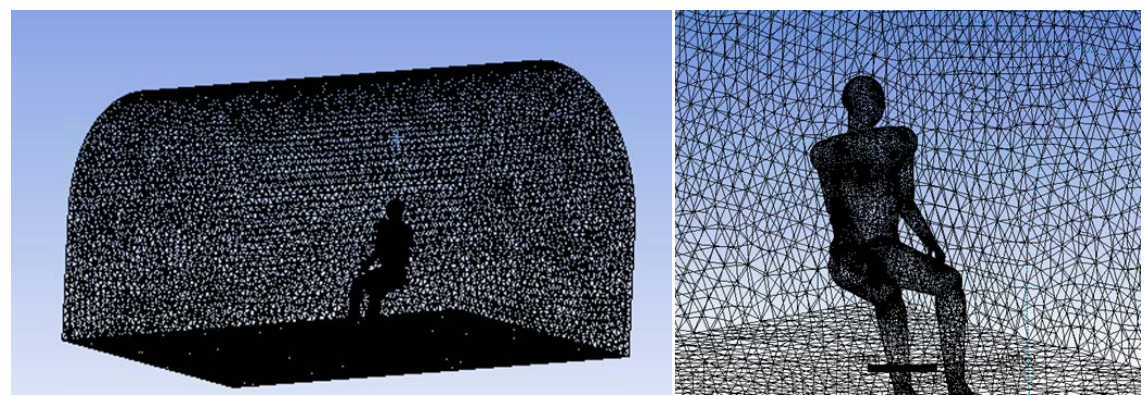

Figure 4. Unstructured mesh with 1.9 million elements and a minimum element size of $0.3 \mathrm{~mm}$.

of this study has been considered an average value of $0.5 \mathrm{~ms}^{-1}$ which is on the lower end of the scale for the value of the velocity of a single breath, although for a complete and more accurate result, rather than the velocity, the frequency should be considered [13].

Thus, the simulation setup for the present study can be summarised as a study of the transport of droplets exhaled from a single breath conducted using a transient air flow condition and a time step $0.001 \mathrm{~s}$ for a total time of $20 \mathrm{~s}$. The total time of $20 \mathrm{~s}$ was chosen as it was the maximum duration that it took the droplets to completely disperse within the cabin area of $10 \mathrm{~m}^{2}$. The choice of the element and time step size ensured that the Courant number had a value of 0.24 , which ensured a stable simulation. In transient simulations, to ensure stability of the simulations, the Courant number should be kept below 1 [14].

\section{Results and Discussions}

In this section, results are presented in a natural logical order depicting the droplet dispersal from a single breath from the index patient in $2 \mathrm{D}$ along the $\mathrm{X}-\mathrm{Y}$ axis following which results are presented in an isometric 3D view. Results are presented mainly to identify the path of the droplet dispersal and their probability of reaching other passengers within the cabin.

The dispersal of droplets and their subsequent mixing with the cabin bulk air flow can be visualised in Figure 5. The figure is oriented along the X-Y axis and presents a side view of the droplet dispersal. The pathlines for the droplet dispersal are visualised at the end of a $20 \mathrm{~s}$ time duration by which point the droplets are completely dispersed within the cabin area. To ensure better visualisation of droplets, the authors considered 600 droplets instead of the average 525 droplets with each droplet having a size of $100 \mu \mathrm{m}$. 


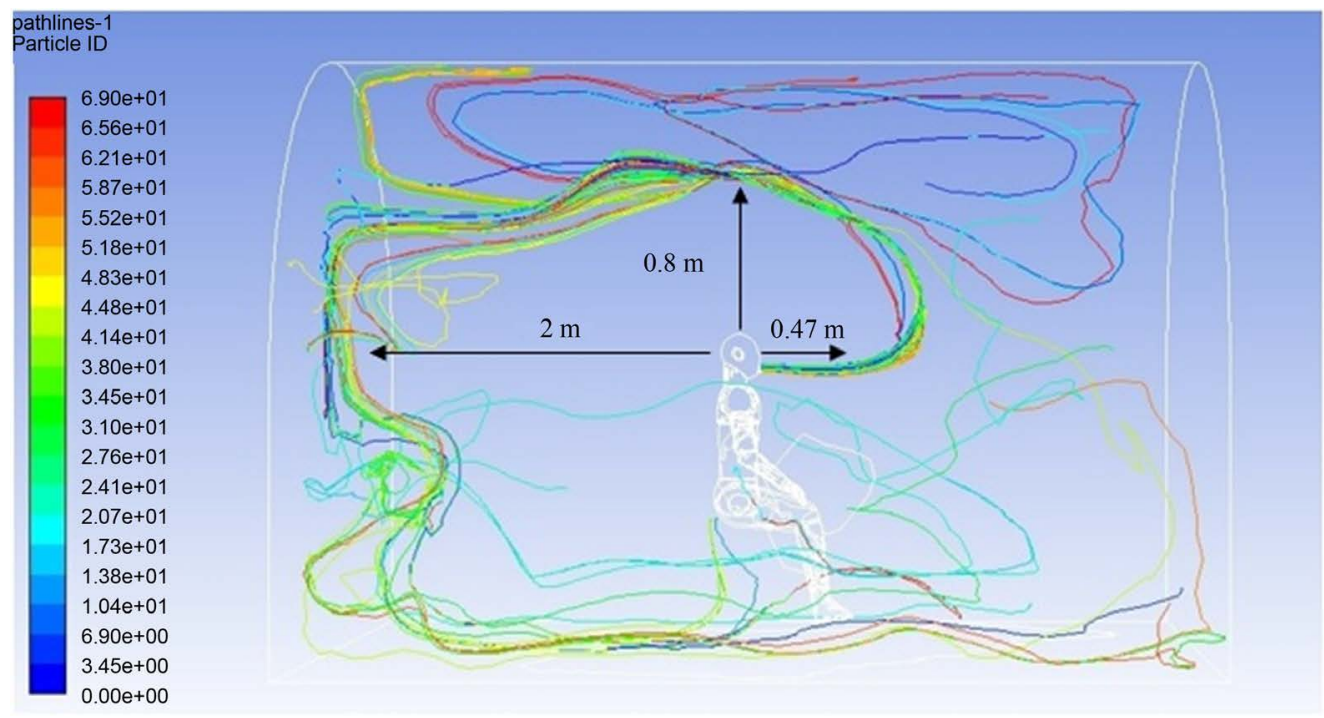

Figure 5. 2D droplet penetration and dispersal in the cabin captured after $20 \mathrm{~s}$ of a single breath.

An isometric view of the droplet dispersal at the end of $20 \mathrm{~s}$ can be observed in Figure 6. Droplets are visualised and tracked through individual "Particle ID's". The pathlines of the droplets indicate a wide dispersal of the droplets amongst the extremities of the cabin. The presence of a single breath as well as the existing air flow within the cabin causes distribution of the droplets within the entire $10 \mathrm{~m}^{2}$ area of the simulated section of the aircraft cabin. Figure 5 indicates that after the index patient exhales, the droplets penetrate about $0.47 \mathrm{~m}$ axially forward before being impacted by the bulk airflow within the cabin. After $0.47 \mathrm{~m}$, the recirculating air current picks up the droplets and causes their dispersal within in the aircraft cabin. The dispersal pattern closely matches existing data presented by Gupta et al. (shown in Figure 1) after $20 \mathrm{~s}$ [7] highlighting the accuracy of the simulation setup as well as the results.

From the pathlines, it can also be observed that the majority of the risk exists to passengers sitting immediately behind the index patient. Approximately $75 \%$ of droplets from the index patient disperse to the area behind the patient. As this forms a majority of the droplets that an average human breathes out in a single breath, the chances of droplet transfer from the index patient to the passengers sitting behind the patient increase dramatically. These droplets also tend to have a high concentration around the facial region of the passenger sitting $2 \mathrm{~m}$ behind the index patient potentially increasing the chance of droplet inhalation. The impact to passengers sitting in front of the index patient appears to be minimal beyond a distance of $0.47 \mathrm{~m}$ from the index patient.

It is also interesting to note that most of the droplets disperse axially behind the mannequin and only disperse transversally at the very back of the cabin area in question (shown in Figure 6). From the pathlines, it can be concluded that passengers sitting transversally away from the index patient are less prone to inhaling the droplets from the index patient. This could potentially result in a lower chance of infection. Thus, the droplet dispersal indicates that passengers 


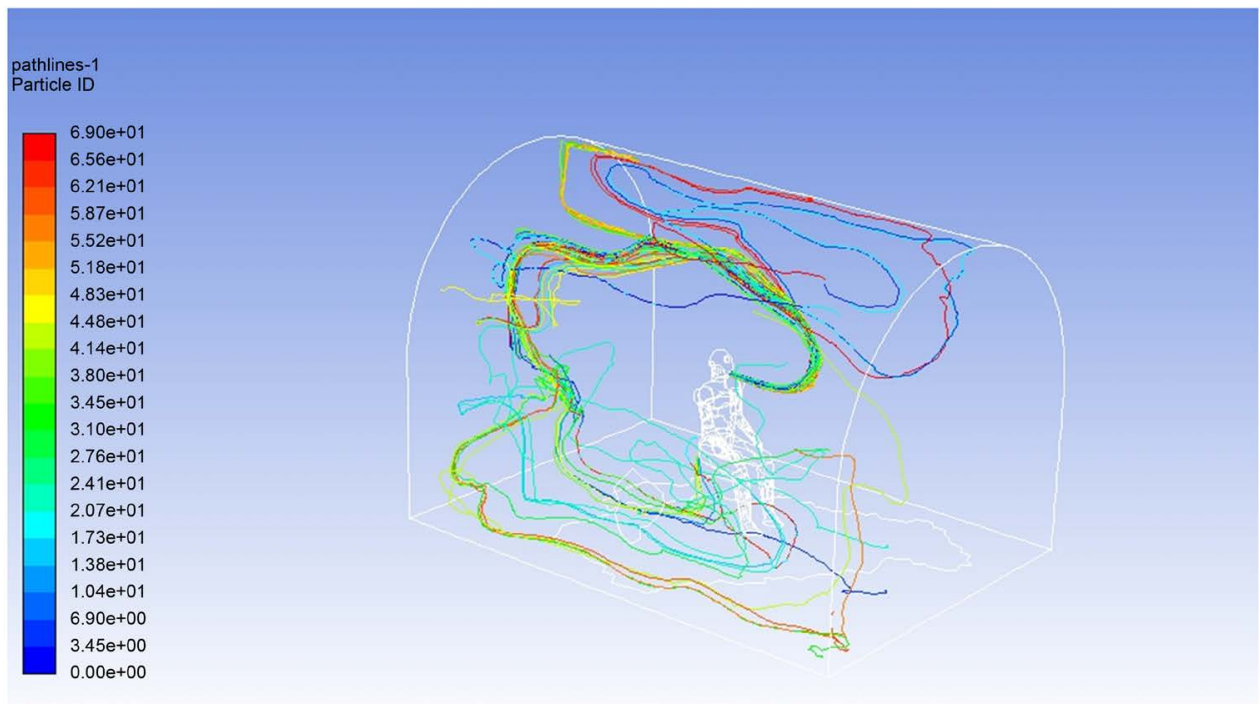

Figure 6. Isometric view of droplet dispersal within the aircraft cabin after $20 \mathrm{~s}$ of a single breath.

within a proximity of $2 \mathrm{~m}$ axially behind the index patient have an enhanced risk of infection through droplet inhalation.

Modern aircraft ventilation systems are designed to use 50\% outside air, and $50 \%$ recirculated air to maintain a more acceptable relative humidity level, and to increase the fuel efficiency [8]. The presence of recirculated air in most scenarios can be a medium for pathogenic transfer. However, to clean all airborne particulate matters and pathogens, the high-efficiency particulate air (HEPA) filters are usually used in modern aircraft. The HEPA filters have a minimum efficiency of $99.97 \%$ for $0.3 \mu \mathrm{m}$ particles when properly installed and maintained [15]. Existing studies indicate that the recirculation of aircraft cabin air does not increase the occupants' risk of being infected by upper respiratory track infections (URIs) on modern commercial aircraft [16]. However, cabin air is recycled 20 - 30 times per hour resulting in a recycling rate of $2-3 \mathrm{~min}$ [17], whereas the droplet dispersal takes only $20 \mathrm{~s}$ to spread within a $10 \mathrm{~m}^{2}$ area. As a result, droplet dispersal still possesses a risk with an aircraft cabin.

Mitigating actions are necessary to reduce the potential risk of airborne infections in an aircraft cabin. Face mask protection has been found to be $45 \%$ in a pseudo-steady environment [18]. Thus, in the present study, the use of a face mask could potentially reduce droplet dispersal by $45 \%$. For air travel to return to normal in the future, several mitigating actions are required by airline companies which are beyond the purview of this paper.

\section{Conclusions}

In this paper, the authors performed a numerical simulation of droplet dispersal within an aircraft cabin. An index patient/mannequin of average human height was modeled and placed inside a designated cabin area. Droplet dispersal due to a single breath was simulated.

Preliminary results in this paper indicate that within $20 \mathrm{~s}$, droplets exhaled 
due to a single breath, mix with the bulk air flow within the cabin and can disperse up to a distance of $2 \mathrm{~m}$ in the axial direction. Passengers sitting behind the index patient are potentially susceptible to this droplet dispersal. The impact due to cross flow dispersal in the transverse direction is minimal.

As the bulk air within the aircraft is recycled every $2-3 \mathrm{~min}$, a dispersal time of $20 \mathrm{~s}$ can be potentially infectious. As this paper is a purely academic exercise to track droplet dispersal, further study is required to understand impact of droplet dispersal within the cabin and the probability of infectious droplets being inhaled by passengers.

\section{Disclaimer}

The authors have presented this study as a purely academic exercise to track droplet dispersal. Results presented herein should not be taken as evidence of any pathogenic spread within an aircraft cabin. The impact and the possibility of pathogenic spread are beyond the purview of the authors' technical expertise.

\section{Acknowledgements}

The authors would like to acknowledge that the financial support for this research was provided by the New Researchers Grant (NRG) from the University of Nottingham Ningbo China.

\section{Conflicts of Interest}

The authors declare no conflicts of interest regarding the publication of this paper.

\section{References}

[1] (2019) Worldwide Market Forecast. 2019-2038. http://www.jadc.jp/files/topics/143_ext_01_en_0.pdf

[2] Global Air Passenger Traffic Plunges 94.3\% in April Due to Covid-19: IATA. https://www.bloombergquint.com/business/global-air-passenger-traffic-plunges-943-in-april-iata

[3] Airlines and COVID-19-KPMG Global. https://home.kpmg/xx/en/home/insights/2020/04/airlines-and-covid-19.html

[4] Tian, Z.F., Tu, J.Y. and Yeoh, G.H. (2007) CFD Studies of Indoor Airflow and Contaminant Particle Transportation. Particulate Science and Technology, 25, 555-570. https://doi.org/10.1080/02726350701492728

[5] Qian, H., Li, Y., Nielsen, P.V. and Huang, X. (2009) Spatial Distribution of Infection Risk of SARS Transmission in a Hospital Ward. Building and Environment, 44, 1651-1658. https://doi.org/10.1016/j.buildenv.2008.11.002

[6] Zhang, T., Chen, Q.Y. and Lin, C.H. (2007) Optimal Sensor Placement for Airborne Contaminant Detection in an Aircraft Cabin. HVAC \& R Research, 13, 683-696. https://doi.org/10.1080/10789669.2007.10390980

[7] Gupta, J.K., Lin, C.-H. and Chen, Q.Y. (2011) Transport of Expiratory Droplets in an Aircraft Cabin. Indoor Air, 21, 3-11. https://doi.org/10.1111/j.1600-0668.2010.00676.x 
[8] Grigg, H.R. and Stewart, R.W. (1963) Turbulent Diffusion in a Stratified Fluid. Journal Fluid Mechanics, 15, 174-186. https://doi.org/10.1017/S0022112063000161

[9] Xie, X., Li, Y., Chwang, A.T.Y., Ho, P.L. and Seto, W.H. (2007) How far Droplets Can Move in Indoor Environments-Revisiting the Wells Evaporation-Falling Curve. Indoor Air, 17, 211-225. https://doi.org/10.1111/j.1600-0668.2007.00469.x

[10] Tips \& Tricks: Convergence and Mesh Independence Study | Computational Fluid Dynamics (CFD) Blog_LEAP Australia \& New Zealand. (2012).

https://www.computationalfluiddynamics.com.au/convergence-and-mesh-indepen dent-study/

[11] Gilani, S., Montazeri, H. and Blocken, B. (2016) CFD Simulation of Stratified Indoor Environment in Displacement Ventilation: Validation and Sensitivity Analysis. Build and Environment, 95, 299-313. https://doi.org/10.1016/j.buildenv.2015.09.010

[12] Ranz, W.E. and Marshall, W.R. (1952) Evaporation from Drops. Chemical Engineering Progress, 48, 141-146.

http://dns2.asia.edu.tw/ ysho/YSHO-English/1000\%20CE/PDF/Che\%20Eng\%20Pr o48,\%20141.pdf

[13] Tang, J.W., Nicolle, A.D., Klettner, C.A., Pantelic, J., Wang, L., Suhaimi, A.B., Tan, A.Y.L., Ong, G.W.X., Su, R., Sekhar, C., Cheong, D.D.W. and Tham, K.W. (2013) Airflow Dynamics of Human Jets: Sneezing and Breathing-Potential Sources of Infectious Aerosols. PLoS ONE, 8, e59970. https://doi.org/10.1371/journal.pone.0059970

[14] Caminha, G. (2019) The CFL Condition and How to Choose Your Timestep Size | SimScale. https://www.simscale.com/blog/2017/08/cfl-condition/

[15] McFadden, E.R., Pichurko, B.M., Bowman, H.F., Ingenito, E., Burns, S., Dowling, N. and Solway, J. (1985) Thermal Mapping of the Airways in Humans. Journal of Applied Physiology, 58, 564-570. https://doi.org/10.1152/jappl.1985.58.2.564

[16] Zitter, J.N., Mazonson, P.D., Miller, D.P., Hulley, S.B. and Balmes, J.R. (2002) Aircraft Cabin Air Recirculation and Symptoms of the Common Cold. The Journal of the American Medical Association, 288, 483-486.

https://doi.org/10.1001/jama.288.4.483

[17] (2018) Cabin Air Quality-Risk of Communicable Diseases Transmission. http://www.iata.org/whatwedo/safety/health/Pages/diseases.aspx

[18] Lai, A.C.K., Poon, C.K.M. and Cheung, A.C.T. (2012) Effectiveness of Facemasks to Reduce Exposure Hazards for Airborne Infections among General Populations. The Royal Society, Interface, 9, 938-948. https://doi.org/10.1098/rsif.2011.0537 